\title{
Theoretical Prediction of Negative Energy Specific to the Electron
}

\author{
Koshun Suto \\ Chudai-Ji Temple, Isesaki, Japan \\ Email: Koshun_suto129@mbr.nifty.com
}

How to cite this paper: Suto, K. (2020) Theoretical Prediction of Negative Energy Specific to the Electron. Journal of Modern Physics, 11, 712-724.

https://doi.org/10.4236/jmp.2020.115046

Received: April 9, 2020

Accepted: May 15, 2020

Published: May 18, 2020

Copyright $\odot 2020$ by author(s) and Scientific Research Publishing Inc. This work is licensed under the Creative Commons Attribution International License (CC BY 4.0).

http://creativecommons.org/licenses/by/4.0/

\begin{abstract}
If an electron emits all of its rest mass energy $m_{\mathrm{e}} c^{2}$, the relativistic energy of the electron will become zero. According to the special theory of relativity, an electron whose relativistic energy is zero does not have photon energy. In this paper, however, an electron is regarded as having photon energy $m_{\mathrm{e}} c^{2}$ and negative energy $-m_{\mathrm{e}} c^{2}$, even when its relativistic energy is zero. The state where relativistic energy is zero is achieved due to the positive energy and negative energy canceling each other out. Relativistic energy becomes zero for an electron in a hydrogen atom when the principle quantum number $n$ is zero. The author has already pointed out the existence of an energy level with $n=0$. If this model is used, it is possible for an electron in the state with $n=0$ to emit additional photons, and transition to negative energy levels. The existence of negative energy specific to the electron has previously been nothing more than a conjecture. However, this paper aims to theoretically show the existence of negative energy based on a discussion using an ellipse. The results show that the electron has latent negative energy.
\end{abstract}

\section{Keywords}

Energy-Momentum Relationship in a Hydrogen Atom, Negative Energy Specific to the Electron, Relativistic Energy, Dark Matter

\section{Introduction}

The most important conclusion derived from the special theory of relativity (STR) is the equivalence of inertial mass and energy [1]. Energy in all its forms has inertial mass [2]. To put it another way, all changes in the energy of an object $\Delta E$ correspond to changes in the object's inertial mass $\Delta m$ [3]. Einstein expressed these as follows. 


$$
\begin{aligned}
E & =m c^{2} . \\
\Delta E & =c^{2} \Delta m .
\end{aligned}
$$

In this paper, let us review the energy-momentum relationship of Einstein using a textbook [4].

Now, in classical mechanics, an increase in kinetic energy corresponds to work done by an external force. That is,

$$
\mathrm{d} E=F \mathrm{~d} x=\frac{\mathrm{d} p}{\mathrm{~d} t} \mathrm{~d} x .
$$

If $v=\mathrm{d} x / \mathrm{d} t$ is used here, Equation (3) can be written as follows.

$$
\mathrm{d} E=v \mathrm{~d} p .
$$

The following relationship also holds in classical mechanics.

$$
m=\frac{p}{v} .
$$

In the textbook of French, the following equation is obtained by combining Equation (1) and Equation (5).

$$
E=\frac{c^{2} p}{v}
$$

Next, if the right-hand sides of Equation (4) and Equation (6), and the corresponding left hand sides, are multiplied together,

$$
E \mathrm{~d} E=c^{2} p \mathrm{~d} p \text {. }
$$

Integrating this,

$$
E^{2}=c^{2} p^{2}+E_{0}^{2} .
$$

Equation (8) can also be expressed as follows.

$$
\left(m c^{2}\right)^{2}=c^{2} p^{2}+\left(m_{0} c^{2}\right)^{2} .
$$

here $m_{0}$ is rest mass and $m$ is relativistic mass. Equation (8) is the energy-momentum relationship of Einstein that holds in an isolated system in free space.

If an object is at rest, $p=0$ and thus Equation (8) is as follows.

$$
E=m_{0} c^{2} .
$$

However, if we are satisfied with Equation (10) only, then the deeper meaning of the theory of relativity is lost. Typically, momentum is not zero. In that case, Equation (1) is used.

Equation (1) includes Equation (10) as a special case. In this paper, the energy in Equation (1) becomes important when dealing with the relativistic energy of the hydrogen atom.

Now, what sort of relation holds in the case of an electron in a hydrogen atom?

Let's consider a situation where an electron at rest in free space is taken into a hydrogen atom due to the electrostatic attraction of the atomic nucleus (proton). At this time, the electron emits a photon and acquires kinetic energy. The in- 
crease in kinetic energy of the electron corresponds to the work done with respect to the outside. This situation is the opposite of Equation (3), and thus Equation (3) must be rewritten as follows.

$$
\mathrm{d} E=-F \mathrm{~d} x=-\frac{\mathrm{d} p}{\mathrm{~d} t} \mathrm{~d} x .
$$

From this, we obtain not Equation (4) but rather

$$
\mathrm{d} E=-v \mathrm{~d} p .
$$

Multiplying in the same way the right-hand sides of Equation (6) and Equation (12), and the corresponding left hand sides,

$$
E \mathrm{~d} E=-c^{2} p \mathrm{~d} p .
$$

And integrating,

$$
E^{2}=-c^{2} p^{2}+E_{0}^{2} .
$$

Equation (14) can also be expressed as follows [5].

$$
\left(m_{n} c^{2}\right)^{2}=-c^{2} p_{n}^{2}+\left(m_{\mathrm{e}} c^{2}\right)^{2}, \quad m_{n}<m_{\mathrm{e}} .
$$

Equation (15) is an energy-momentum relationship applicable to an electron in a hydrogen atom which has potential energy.

here, $m_{\mathrm{e}} c^{2}$ is the rest mass energy of the electron, and $m_{n} c^{2}$ is the relativistic energy of the electron. The subscript $n$ is the principal quantum number.

Incidentally, the author has previously conjectured that the electron has latent negative energy in ref. [6]. However, since then no papers have been published developing the author's conjecture. Therefore, this paper aims to theoretically predict the existence of this negative energy.

\section{Relativistic Correction of the Bohr Energy Levels}

Past attempts to relativistically expand the energy levels of the hydrogen atom derived by Bohr have taken Equation (8) as their point of departure [7] [8] [9] [10]. However, this is a mistake.

The equation which treats an electron in a hydrogen atom relativistically is not the Dirac equation satisfying Equation (8). It must be another equation satisfying Equation (14). The author has already derived this equation [11].

The energy levels derived by Bohr are given by the following formulas [12] [13].

$$
\begin{aligned}
E_{\mathrm{BO}, n} & =-\frac{1}{2}\left(\frac{1}{4 \pi \varepsilon_{0}}\right)^{2} \frac{m_{\mathrm{e}} e^{4}}{\hbar^{2}} \cdot \frac{1}{n^{2}} \\
E_{\mathrm{BO}, n} & =-\frac{\alpha^{2} m_{\mathrm{e}} c^{2}}{2 n^{2}}, \quad n=1,2, \cdots .
\end{aligned}
$$

here, $E_{\mathrm{BO}, n}$ signifies the energy levels derived by Bohr. Also, $\alpha$ is the fine-structure constant, and is defined as follows.

$$
\alpha=\frac{e^{2}}{4 \pi \varepsilon_{0} \hbar c}=7.2973525693 \times 10^{-3} .
$$


In Bohr's theory, the energy when the electron has been pulled away from the atomic nucleus, and is stationary at an infinite distance, is set to be zero. However, the true energy in this state is $m_{\mathrm{e}} c^{2}$. The relativistic energy $m_{n} c^{2}$ in Equation (15) indicates the energy with an absolute scale.

Incidentally, Einstein and Sommerfeld defined kinetic energy as the difference between the total energy and rest mass energy of an object [14]. That is,

$$
K=m c^{2}-m_{0} c^{2}=m_{0} c^{2}\left[\frac{1}{\left(1-v^{2} / c^{2}\right)^{1 / 2}}-1\right] .
$$

If this definition is also used for the kinetic energy of an electron in a hydrogen atom, then the energy levels of the hydrogen atom can be defined as follows.

$$
E_{\mathrm{re}, n}=-K_{\mathrm{re}, n}=m_{n} c^{2}-m_{\mathrm{e}} c^{2} .
$$

here, $K_{\mathrm{re}, n}$ is the relativistic kinetic energy of an electron [15]. However, it is not appropriate to call $E_{\mathrm{re}, n}$ relativistic energy. The relativistic energy of an electron has already been defined by Equation (15). $E_{\mathrm{re}, n}$ gives energy levels which correct the Bohr energy levels (16) from the perspective of the theory of relativity. However, it should be noted that the term "theory of relativity" is applied not in the sense of Equation (9) but in the sense of Equation (15).

Incidentally, the following formula can be derived from Equation (9).

$$
m=\frac{m_{0}}{\left(1-v^{2} / c^{2}\right)^{1 / 2}} .
$$

Using similar mathematics, the following formula can be derived from Equation (15) [16].

$$
m_{n}=\frac{m_{\mathrm{e}}}{\left(1+v_{n}^{2} / c^{2}\right)^{1 / 2}}, \quad m_{n}<m_{\mathrm{e}} .
$$

An electron in a hydrogen atom becomes lighter in mass as it increases in speed. This is the opposite of the prediction of STR.

Next, Equation (21) is rewritten using the following relationship derived in ref. [15].

$$
\frac{v_{n}}{c}=\frac{\alpha}{n}
$$

when that is done,

$$
m_{n}=\frac{m_{\mathrm{e}}}{\left(1+\alpha^{2} / n^{2}\right)^{1 / 2}} .
$$

Hence the energy levels in Equation (19) become as follows.

$$
E_{\mathrm{re}, n}=m_{n} c^{2}-m_{\mathrm{e}} c^{2}=m_{\mathrm{e}} c^{2}\left[\left(\frac{n^{2}}{n^{2}+\alpha^{2}}\right)^{1 / 2}-1\right], n=0,1,2, \cdots
$$

To simplify the discussion in this paper, the only quantum number addressed 
is $n$.

Next, when the part of Equation (24) in parentheses is expressed as a Taylor expansion,

$$
\begin{gathered}
E_{\mathrm{re}, n} \approx m_{\mathrm{e}} c^{2}\left[\left(1-\frac{\alpha^{2}}{2 n^{2}}+\frac{3 \alpha^{4}}{8 n^{4}}-\frac{5 \alpha^{6}}{16 n^{6}}\right)-1\right] \\
E_{\mathrm{re}, n} \approx-\frac{\alpha^{2} m_{\mathrm{e}} c^{2}}{2 n^{2}} .
\end{gathered}
$$

It is evident from this that the Bohr energy levels (16b) are an approximation of Equation (24).

Incidentally, it was once pointed out by Dirac that Equation (8) has a negative solution [17]. In the same way, the author has pointed out that Equation (15) has a negative solution [18]. The mass of an electron at negative energy levels becomes negative.

In the current universe, there is thought to exist a tremendous mass whose true nature is unknown (an unknown source of gravity). The author has presented matter formed from an electron with negative mass and a proton (atomic nucleus) with positive mass as a strong candidate for this unknown matter, i.e., dark matter [19] [20] [21] [22].

Now, if the negative solution of Equation (15) is also incorporated, then the relativistic energy $E_{\mathrm{abre}, n}^{ \pm}$of a hydrogen atom can be written as follows.

$$
E_{\mathrm{abre}, n}^{ \pm}= \pm m_{n} c^{2}= \pm m_{\mathrm{e}} c^{2} \mp K_{\mathrm{re}, n}= \pm m_{\mathrm{e}} c^{2}\left(\frac{n^{2}}{n^{2}+\alpha^{2}}\right)^{1 / 2}, n=0,1,2, \cdots .
$$

The $E_{\mathrm{abre}, n}$ newly introduced here has a long subscript. However, the reason for this is to distinguish from $E_{\mathrm{re}, n}$ in Equation (19). $E_{\mathrm{abre}, n}$ is relativistic energy, but this is also energy expressed with an absolute scale. The "ab" in the subscript stands for "absolute," while "re" standard for "relativistic." If the above points are kept in mind, then there is no problem in abbreviating $E_{\mathrm{abre}, n}$ as $E_{\mathrm{ab}, n}$.

\section{Equation (15) Derived from an Ellipse}

In this section, Equation (15) is derived using an ellipse.

First, consider the Cartesian coordinate system $\mathrm{O}-x y$. Letting $\mathrm{F}$ and $\mathrm{F}$ ' be the points $x= \pm f$, an ellipse is drawn taking those 2 points as foci (Figure 1 ).

Let $\mathrm{A}$ and $\mathrm{A}^{\prime}$ be the points where the ellipse intersects the $x$-axis, and let $\mathrm{B}$ and $\mathrm{B}^{\prime}$ be the points where the ellipse intersects the $y$-axis. Also, let $2 a$ be the length of the line segment $\overline{\mathrm{AA}^{\prime}}, 2 b$ be the length of the line segment $\overline{\mathrm{BB}^{\prime}}$, and $2 f$ be the length of the line segment $\overline{\mathrm{FF}^{\prime}}$.

The eccentricity of the ellipse in this case is defined as follows.

$$
\varepsilon=\frac{f}{a} \text {. }
$$

The eccentricity of the ellipse can also be expressed using the following formula. 


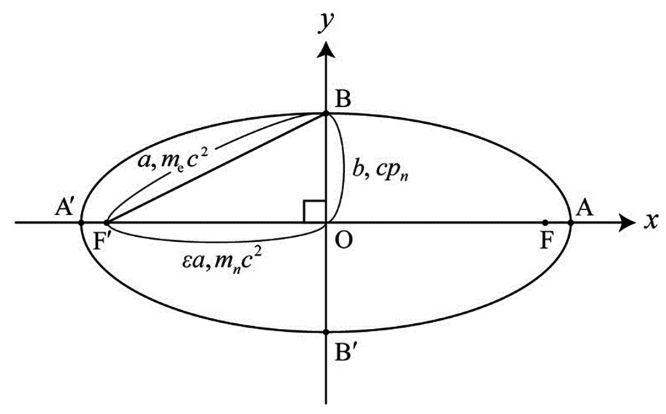

Figure 1. First, the energy $m_{\mathrm{e}} c^{2}$ is taken to correspond to the line segment $\overline{\mathrm{OA}}$, and then Equation (31) is assumed. Equation (15) can be derived if the Pythagorean theorem is applied to the right triangle $\mathrm{OBF}^{\prime}$.

$$
\varepsilon=\left(1-\frac{b^{2}}{a^{2}}\right)^{1 / 2}
$$

The following equation can be derived from Equation (28).

$$
b=a\left(1-\varepsilon^{2}\right)^{1 / 2}
$$

here, the line segment $\overline{\mathrm{OA}}$ is taken to correspond to the energy $m_{\mathrm{e}} c^{2}$. Let us express this as follows.

$$
a=m_{\mathrm{e}} c^{2} .
$$

Also, assume the following relation.

$$
\varepsilon=\left(\frac{n^{2}}{n^{2}+\alpha^{2}}\right)^{1 / 2}
$$

Taking Equation (30) and Equation (31) into account, $b$ can be expressed with the following equations.

$$
\begin{aligned}
b & =m_{\mathrm{e}} c^{2}\left[1-\left(\frac{n^{2}}{n^{2}+\alpha^{2}}\right)\right]^{1 / 2} \\
& =m_{\mathrm{e}} c^{2}\left(\frac{\alpha^{2}}{n^{2}+\alpha^{2}}\right)^{1 / 2} .
\end{aligned}
$$

Incidentally, if the length of the hypotenuse $\mathrm{AB}$ is taken to be $c$ in the right triangle AOB in Figure 1, then the Pythagorean theorem below holds for the lengths of the three sides of the right triangle.

$$
c^{2}=a^{2}+b^{2} \text {. }
$$

Next, the Pythagorean theorem is applied to the right triangle $\mathrm{OBF}^{\prime}$. At that time, taking into account that $f=a \varepsilon$, we obtain

$$
(\varepsilon a)^{2}+b^{2}=\left(\frac{n^{2}}{n^{2}+\alpha^{2}}\right)\left(m_{\mathrm{e}} c^{2}\right)^{2}+\left(\frac{\alpha^{2}}{n^{2}+\alpha^{2}}\right)\left(m_{\mathrm{e}} c^{2}\right)^{2}=\left(m_{\mathrm{e}} c^{2}\right)^{2} .
$$

here, if Equation (23) and Equation (22) are also taken into consideration, then the equation in Equation (34) can be expressed as follows. 
$\left(\frac{\alpha^{2}}{n^{2}+\alpha^{2}}\right)\left(m_{\mathrm{e}} c^{2}\right)^{2}=\left(\frac{\alpha^{2}}{n^{2}+\alpha^{2}}\right) m_{n}^{2}\left(\frac{n^{2}+\alpha^{2}}{n^{2}}\right) c^{2} \cdot c^{2}=\frac{\alpha^{2}}{n^{2}} \cdot m_{n}^{2} \frac{n^{2} v_{n}^{2}}{\alpha^{2}} c^{2}=c^{2} p_{n}^{2}$

Substituting this result for Equation (35) into Equation (34),

$$
(\varepsilon a)^{2}+b^{2}=\left(m_{n} c^{2}\right)^{2}+c^{2} p_{n}^{2}=\left(m_{\mathrm{e}} c^{2}\right)^{2} .
$$

when $m_{\mathrm{e}} c^{2}$ is taken to correspond to the line segment $\overline{\mathrm{OA}}$, and Equation (31) is assumed, then Equation (15) can be derived from the right triangle OBF' Also, from Equation (30) and Equation (32b),

$$
\frac{b}{a}=\left(\frac{\alpha^{2}}{n^{2}+\alpha^{2}}\right)^{1 / 2} \text {. }
$$

when Equation (37) is substituted for $\alpha$ in Equation (17), it is evident that the ellipse that should actually be drawn is far flatter than that in Figure 1. However, to make the diagram easier to read, the ellipse is drawn with an elongated $y$-axis in this paper. (The segment $\overline{\mathrm{AF}}$ was also drawn longer than it actually is for the same reason.)

Incidentally, the author has previously derived the following relation in ref. [21].

$$
\frac{r_{n}^{-}}{r_{n}^{+}}=\frac{\left(n^{2}+\alpha^{2}\right)^{1 / 2}-n}{\left(n^{2}+\alpha^{2}\right)^{1 / 2}+n}=\frac{m_{\mathrm{e}}-m_{n}}{m_{\mathrm{e}}+m_{n}} .
$$

However, when considered using an ellipse, Equation (38) can be expanded as follows (Figure 2).

$$
\frac{\overline{\mathrm{AF}}}{\overline{\mathrm{AF}^{\prime}}}=\frac{a-f}{a+f}=\frac{1-\varepsilon}{1+\varepsilon}=\frac{r_{n}^{-}}{r_{n}^{+}}=\frac{\left(n^{2}+\alpha^{2}\right)^{1 / 2}-n}{\left(n^{2}+\alpha^{2}\right)^{1 / 2}+n}=\frac{\left(m_{\mathrm{e}}-m_{n}\right) c^{2}}{\left(m_{\mathrm{e}}+m_{n}\right) c^{2}} .
$$

Also, the following relation can be obtained from Figure 1.

$$
\frac{\overline{\mathrm{FF}^{\prime}}}{\overline{\mathrm{AA}^{\prime}}}=\varepsilon=\frac{\left(r_{n}^{+}+r_{n}^{-}\right)-2 r_{n}^{-}}{r_{n}^{+}+r_{n}^{-}}=\frac{r_{n}^{+}-r_{n}^{-}}{r_{n}^{+}+r_{n}^{-}}=\frac{m_{n}}{m_{\mathrm{e}}}=\left(\frac{n^{2}}{n^{2}+\alpha^{2}}\right)^{1 / 2} .
$$

In Equation (39), $r_{n}^{+}$was taken to correspond to the line segment $\overline{\mathrm{AF}^{\prime}}$, and $r_{n}^{-}$was taken to correspond to the line segment $\overline{\mathrm{AF}}$. Also, if $m_{\mathrm{e}} c^{2}$ is taken to correspond to the line segment $\overline{\mathrm{OA}}$, based on Equation (40), then $2 m_{\mathrm{e}} c^{2}$ corresponds to line segment $\overline{\mathrm{AA}^{\prime}}$. At this time, $\left(m_{\mathrm{e}}-m_{n}\right) c^{2}$ corresponds to line segment $\overline{\mathrm{AF}}$, and $\left(m_{\mathrm{e}}+m_{n}\right) c^{2}$ corresponds to the line segment $\overline{\mathrm{AF}^{\prime}}$.

However, this does not mean that line segment length expresses the absolute value of a physical quantity, so caution is necessary. Also the orbital radii $r_{n}^{+}$ and $r_{n}^{-}$at different energy levels are apparent in the ellipse in Figure 2. This shows that this ellipse is not an illustration of the orbital of a single electron. The discussion thus far is summarized in the following table (Table 1).

here, $r_{\mathrm{e}}$ is the classical electron radius, defined as follows.

$$
r_{\mathrm{e}}=\frac{e^{2}}{4 \pi \varepsilon_{0} m_{\mathrm{e}} c^{2}} .
$$




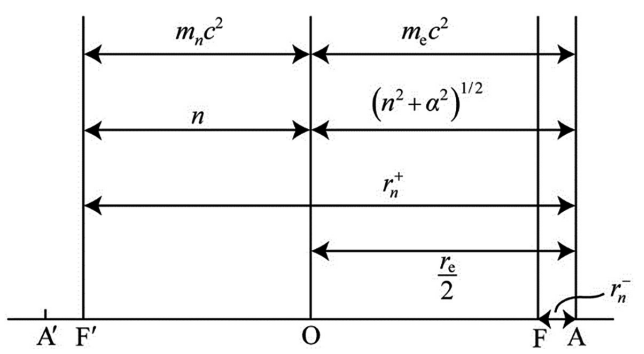

Figure 2. Caution is necessary because the lengths of the line segments in this diagram do not give the absolute value of physical quantities.

Table 1. Electron energy corresponding to each line segment and orbital radius. There are differences between energy ${ }^{\star} 1$ and ${ }^{\star} 2$, and energy ${ }^{\star} 3$ and ${ }^{\star} 4$. These energy differences are discussed in section 4 .

\begin{tabular}{cccc}
\hline Line Segment & $\overline{\mathrm{AF}}$ & $\overline{\mathrm{AO}}$ & $\overline{\mathrm{AF}^{\prime}}$ \\
\hline $\begin{array}{c}\text { Orbital Radius } \\
\text { Corresponding to Line Segment } \\
\text { Relativistic Energy of Electron }\end{array}$ & $r_{n}^{-}$ & $\frac{r_{\mathrm{e}}}{2}$ & $r_{n}^{+}$ \\
Indication using $n$ and $\alpha$ & $\left(m^{2} c^{2}\right.$ & $0 * 3$ & $\left.\alpha^{2}\right)^{1 / 2}-n$ \\
$\begin{array}{c}\text { Energy Corresponding to } \\
\text { Line Segment }\end{array}$ & $\left(m_{\mathrm{e}}-m_{n}\right) c^{2}$ & $\left.m^{2}+\alpha^{2}\right)^{1 / 2}$ & $\left(n^{2}+\alpha^{2}\right)^{1 / 2}+n$ \\
\hline
\end{tabular}

Incidentally, when the electron's relativistic energy $m_{n} c^{2}$ becomes zero, then the value of $E_{\mathrm{re}, n}$ in Equation (19) is

$$
E_{\mathrm{re}, 0}=-m_{\mathrm{e}} c^{2}, \quad m_{n} c^{2}=0 .
$$

Also, the following formula can be derived from the relationship between hydrogen atom energy levels and potential energy.

$$
E_{\mathrm{re}, n}=\frac{1}{2} V\left(r_{n}\right)=-\frac{1}{2} \frac{1}{4 \pi \varepsilon_{0}} \frac{e^{2}}{r_{n}}=-\frac{1}{2} \frac{r_{\mathrm{e}} m_{\mathrm{e}} c^{2}}{r_{n}}=-m_{\mathrm{e}} c^{2}\left(\frac{r_{\mathrm{e}} / 2}{r_{n}}\right) .
$$

here, if Equation (42) and Equation (43) are compared, the radius $r_{0}$ when $n=0$, is

$$
r_{0}=\frac{r_{\mathrm{e}}}{2} .
$$

Incidentally, the electron does not have a definite orbital in quantum mechanics. The author has previously predicted in ref. [6] that the radius of the atomic nucleus of the hydrogen atom (proton) is $r_{\mathrm{e}} / 4$. However, this is not a claim that the atomic nucleus is completely spherical. The meaning is that statistically the atomic nucleus can be regarded as a sphere with radius $r_{\mathrm{e}} / 4$. In the same way, this paper does not assert that an electron with energy $E_{\mathrm{ab}, n}^{+}$revolves in an elliptical orbital with semi-major radius $r_{n}^{+}$. (In quantum mechanics, the Bohr radius $a_{\mathrm{B}}$ of the hydrogen atom signifies the radius where the probability of finding the electron is maximal.) 


\section{Discussion}

1) In Figure 2 and Table 1 , the relativistic energy corresponding to $n$ is $m_{n} c^{2}\left({ }^{*} 1\right)$. The orbital radius $r_{n}^{+}$of an electron with this energy becomes $\left(n^{2}+\alpha^{2}\right)^{1 / 2}+n$ when indicated in terms of $n$ and $\alpha$. However, the energy corresponding to this is $\left(m_{\mathrm{e}}+m_{n}\right) c^{2} \quad\left({ }^{\star} 2\right)$.

There are two types of electron energy in a certain state. How should we think about this? First of all, the following two points are correct.

a) The relativistic energy of an electron with orbital radius of $r_{n}^{+}$is $m_{n} c^{2}$ $\left({ }^{*} 1\right)$.

b) The energy of an electron with orbital radius of $r_{n}^{+}$is $\left(m_{\mathrm{e}}+m_{n}\right) c^{2} \quad\left({ }^{*} 2\right)$.

We must elucidate the true nature of the energy $\left(m_{\mathrm{e}}+m_{n}\right) c^{2}$. The author has previously assumed in ref. [6] that an electron in a state with $n=0$ has photon energy and negative energy. Let's use that as a hint.

Now, taking Figure 2 and the existence of negative energy into account, Table 1 can be expanded as follows (Table 2).

Here, if the photon energy possessed by an electron is expressed as $E_{\mathrm{ph}, n}$, then the relativistic energy of the electron $E_{a b, n}$ defined in Equation (26) can be written as follows.

$$
E_{\mathrm{ab}, n}=E_{\mathrm{ph}, n}-m_{\mathrm{e}} c^{2} .
$$

According to this model, $m_{n} c^{2}\left({ }^{*} 1\right)$ is the relativistic energy of the electron, and $\left(m_{\mathrm{e}}+m_{n}\right) c^{2} \quad\left({ }^{\star} 2\right)$ is the photon energy possessed by the electron.

Incidentally, the relativistic energy of an electron $E_{\mathrm{ab}, n}$ is thought to be what determines the electron's orbital radius. However, in Equation (45), the interpretation is also possible that it is $E_{\mathrm{ph}, n}$ which determined the orbital radius of the electron.

Now, in the state where $E_{\mathrm{ab}}=0$, the photon energy possessed by the electron and the negative energy $-m_{\mathrm{e}} c^{2}$ cancel each other out (the relationship of ${ }^{*} 3,{ }^{*} 4$, and ${ }^{*}$ in Table 2). If this model is used, an electron with $E_{\mathrm{ab}}=0$ can emit additional photons, and transition to negative energy levels.

Table 2. Relationship of the relativistic energy of an electron, and the photon energy possessed by that electron. The relativistic energy of an electron is defined as the sum of the photon energy possessed by that electron, and the negative energy specific to it.

\begin{tabular}{|c|c|c|c|c|c|}
\hline Line Segment & - & $\overline{\mathrm{AF}}, a-f$ & $\overline{\mathrm{AO}}, a$ & $\overline{\mathrm{AF}^{\prime}}, a+f$ & - \\
\hline Eccentricity Indication & - & $(1-\varepsilon) a$ & $a$ & $(1+\varepsilon) a$ & - \\
\hline Orbital Radius & $\frac{r_{\mathrm{e}}}{4}$ & $r_{n}^{-}$ & $\frac{r_{\mathrm{e}}}{2}$ & $r_{n}^{+}$ & $\infty$ \\
\hline Indication using $n$ and $\alpha$ & - & $\left(n^{2}+\alpha^{2}\right)^{1 / 2}-n$ & $\left(n^{2}+\alpha^{2}\right)^{1 / 2}$ & $\left(n^{2}+\alpha^{2}\right)^{1 / 2}+n$ & - \\
\hline $\begin{array}{l}\text { Relativistic Energy of } \\
\text { Electron } E_{\mathrm{ab}, n}, \quad E_{\mathrm{ph}, n}-m_{\mathrm{e}} c^{2}\end{array}$ & $-m_{e} c^{2}$ & $-m_{n} c^{2}$ & $0 * 3$ & $m_{n} c^{2}{ }^{\star} 1$ & $m_{\mathrm{e}} c^{2}{ }^{*} 6$ \\
\hline Photon Energy $E_{\mathrm{ph}, n}$ & 0 & $\left(m_{\mathrm{e}}-m_{n}\right) c^{2}$ & $m_{\mathrm{e}} c^{2} \star_{4}$ & $\left(m_{\mathrm{e}}+m_{n}\right) c^{2} \star_{2}$ & $2 m_{\mathrm{e}} c^{2} *$ \\
\hline Negative Energy $-m_{\mathrm{e}} c^{2}$ & $-m_{\mathrm{e}} c^{2}$ & $-m_{\mathrm{e}} c^{2}$ & $-m_{\mathrm{e}} c^{2} \star_{5}$ & $-m_{\mathrm{e}} c^{2}$ & $-m_{\mathrm{e}} c^{2} * \varepsilon$ \\
\hline
\end{tabular}


Also, according to the STR, an electron has a rest mass energy of $m_{\mathrm{e}} c^{2}$. However, in this paper, it is thought that an electron at rest has photonic energy $2 m_{\mathrm{e}} c^{2}$ and negative energy $-m_{\mathrm{e}} c^{2}$ (the relationship of ${ }^{*} 6,{ }^{*} 7$, and ${ }^{*} 8$ in Table 2). If symmetry is taken into account, it is likely valid to think that the true nature of this negative energy is a photon with negative mass. (However, that does not mean that in this paper it was possible to determine the amount of negative energy to be $-m_{\mathrm{e}} c^{2}$.)

2) The author has previously derived the following formulas in ref. [21].

$$
\begin{aligned}
& r_{n}^{+}=\frac{r_{\mathrm{e}}}{2} \frac{\left(n^{2}+\alpha^{2}\right)^{1 / 2}}{\left(n^{2}+\alpha^{2}\right)^{1 / 2}-n} . \\
& r_{n}^{-}=\frac{r_{\mathrm{e}}}{2} \frac{\left(n^{2}+\alpha^{2}\right)^{1 / 2}}{\left(n^{2}+\alpha^{2}\right)^{1 / 2}+n} .
\end{aligned}
$$

Equation (46) and Equation (47) can be written as follows.

$$
\begin{aligned}
& r_{n}^{+}=\frac{r_{\mathrm{e}}}{2}\left[1+\frac{n}{\left(n^{2}+\alpha^{2}\right)^{1 / 2}-n}\right] . \\
& r_{n}^{-}=\frac{r_{\mathrm{e}}}{2}\left[1-\frac{n}{\left(n^{2}+\alpha^{2}\right)^{1 / 2}+n}\right] .
\end{aligned}
$$

Also, the following equation holds if Equation (39) is taken into account.

$$
\begin{gathered}
r_{n}^{+}=\frac{r_{\mathrm{e}}}{2} \cdot \frac{m_{\mathrm{e}}}{m_{\mathrm{e}}-m_{n}}=\frac{r_{\mathrm{e}}}{2}\left(1+\frac{m_{n}}{m_{\mathrm{e}}-m_{n}}\right) . \\
r_{n}^{-}=\frac{r_{\mathrm{e}}}{2} \cdot \frac{m_{\mathrm{e}}}{m_{\mathrm{e}}+m_{n}}=\frac{r_{\mathrm{e}}}{2}\left(1-\frac{m_{n}}{m_{\mathrm{e}}+m_{n}}\right), \frac{r_{\mathrm{e}}}{4}<r_{\mathrm{e}}^{-} .
\end{gathered}
$$

In addition, if the eccentricity of the ellipse is used,

$$
\begin{aligned}
& r_{n}^{+}=\frac{r_{\mathrm{e}}}{2}\left[1+\frac{n}{\left(n^{2}+\alpha^{2}\right)^{1 / 2}-n}\right]=\frac{r_{\mathrm{e}}}{2}\left(1+\frac{\varepsilon_{n}}{1-\varepsilon_{n}}\right) . \\
& r_{n}^{-}=\frac{r_{\mathrm{e}}}{2}\left[1-\frac{n}{\left(n^{2}+\alpha^{2}\right)^{1 / 2}+n}\right]=\frac{r_{\mathrm{e}}}{2}\left(1-\frac{\varepsilon_{n}}{1+\varepsilon_{n}}\right) .
\end{aligned}
$$

here, if Equation (48) and Equation (49), and Equation (50) and Equation (51) are respectively combined into single formulas, the results are as follows.

$$
\begin{gathered}
r_{n}^{ \pm}=\frac{r_{\mathrm{e}}}{2}\left[1 \pm \frac{n}{\left(n^{2}+\alpha^{2}\right)^{1 / 2} \mp n}\right] . \\
r_{n}^{ \pm}=\frac{r_{\mathrm{e}}}{2}\left(1 \pm \frac{m_{n}}{m_{\mathrm{e}} \mp m_{n}}\right) .
\end{gathered}
$$


The orbital radius of an electron is defined as the distance from the center of the atomic nucleus to the electron. However, according to Equation (54) and Equation (55), the sizes of $r_{n}^{+}$and $r_{n}^{-}$are determined taking $r=r_{\mathrm{e}} / 2$ as a starting point.

This is a discovery that has not been previously stated. $r_{n}^{-}$approaches the atomic nucleus (radius $r_{\mathrm{e}} / 4$ ) as $n$ increases. In quantum mechanics, lower energy is regarded as more stable, but that is incorrect. Actually, the closer the relativistic energy of an electron is to zero, the greater the stability. Therefore, electrons with negative energy are never easily incorporated into the atomic nucleus.

Next, let us investigate the relationship between $r_{n}^{+}$and the Bohr radius of the hydrogen atom. The Bohr radius is given by the following formula.

$$
a_{\mathrm{B}} n^{2}=r_{1} n^{2}=4 \pi \varepsilon_{0} \cdot \frac{\hbar^{2}}{m_{\mathrm{e}} e^{2}} \cdot n^{2}=\frac{r_{\mathrm{e}}}{\alpha^{2}} \cdot n^{2} .
$$

Next, Equation (46) is rewritten as follows.

$$
\begin{aligned}
r_{n}^{+} & =\frac{r_{\mathrm{e}}}{2} \cdot \frac{\left(n^{2}+\alpha^{2}\right)^{1 / 2}}{\left(n^{2}+\alpha^{2}\right)^{1 / 2}-n} \cdot \frac{\left(n^{2}+\alpha^{2}\right)^{1 / 2}+n}{\left(n^{2}+\alpha^{2}\right)^{1 / 2}+n} \\
& =\frac{r_{\mathrm{e}}}{2} \cdot \frac{n^{2}+\alpha^{2}+n^{2}\left(1+\alpha^{2} / n^{2}\right)^{1 / 2}}{\alpha^{2}} .
\end{aligned}
$$

when the part of the numerator in Equation (56b) that is in parentheses is developed as a Taylor expansion,

$$
\left(1+\frac{\alpha^{2}}{n^{2}}\right)^{1 / 2} \approx 1+\frac{\alpha^{2}}{2 n^{2}}-\frac{\alpha^{4}}{8 n^{4}}+\frac{\alpha^{6}}{16 n^{6}} .
$$

If this is substituted into Equation (57b),

$$
r_{n}^{+}=\frac{r_{\mathrm{e}}}{\alpha^{2}} \cdot n^{2}+\frac{r_{\mathrm{e}}}{2}\left(\frac{3}{2}-\frac{\alpha^{2}}{8 n^{2}}\right) \approx \frac{r_{\mathrm{e}}}{\alpha^{2}} \cdot n^{2}=a_{\mathrm{B}} n^{2} .
$$

From this, it is evident that the Bohr radius is an approximate value for Equation (46).

\section{Conclusions}

1) In this paper, the mathematically derived Equation (15) was also derived from a discussion using an ellipse. This paper was also able to theoretically predict the existence of latent negative energy possessed by the electron. The relationship is unclear between this negative energy, and the negative energy (dark energy) whose true nature is unknown, and which is currently thought to exist in large quantities in the universe. Further research will be needed in the future to elucidate that point.

2) The author has believed that the orbital radius of an electron forming a hydrogen atom is determined by the electron's relativistic energy $E_{\mathrm{ab}, n}$. Even this is definitely not a mistake. However, this paper has shown more accurately that 
what determines the orbital radius of the electron is the photon energy $E_{\mathrm{ph}, n}$ possessed by the electron.

Also, the orbital radius of the electron is given as the distance from the center of the atomic nucleus (proton) to the electron. However, this paper has shown that the size of the electron orbital will be determined taking $r=r_{\mathrm{e}} / 2$ as the starting point.

\section{Acknowledgements}

I would like to express my thanks to the staff at ACN Translation Services for their translation assistance. Also, I wish to express my gratitude to Mr. Shimada, H. for drawing figures.

\section{Conflicts of Interest}

The author declares no conflicts of interest regarding the publication of this paper.

\section{References}

[1] Einstein, A. (1961) Relativity. Crown, New York, 43.

[2] Einstein, A. (1946) Technical Journal, 5, 16.

[3] French, A.P. (1968) Special Relativity. W.W. Norton \& Company, New York, London, 19.

[4] French, A.P. (1968) Special Relativity. W.W. Norton \& Company, New York, London, 21.

[5] Suto, K. (2011) Physics Essays, 24, 301-307. https://doi.org/10.4006/1.3583810

[6] Suto, K. (2018) Applied Physics Research, 10, 93-101. https://doi.org/10.5539/apr.v10n4p93

[7] Kraft, D.W. (1974) American Journal of Physics, 42, 837-839. https://doi.org/10.1119/1.1987875

[8] Cushing, J.T. (1970) American Journal of Physics, 38, 1145. https://doi.org/10.1119/1.1976568

[9] Von Baeyer, H.C. (1975) Physical Review D, 12, 3086. https://doi.org/10.1103/PhysRevD.12.3086

[10] Terzis, A.F. (2008) European Journal of Physics, 29, 735-743. https://doi.org/10.1088/0143-0807/29/4/008

[11] Suto, K. (2018) Applied Physics Research, 10, 102-108. https://doi.org/10.5539/apr.v10n6p102

[12] Bohr, N. (1913) Philosophical Magazine, 26, 1. https://doi.org/10.1080/14786441308634955

[13] Bohr, N. (1952) Collected Works Vol. 2. North-Holland, Amsterdam, 136.

[14] Sommerfeld, A. (1923) Atomic Structure and Spectral Lines. Methuen \& Co. Ltd., London, 528.

[15] Suto, K. (2019) Applied Physics Research, 11, 19-34. https://doi.org/10.5539/apr.v11n1p19

[16] Suto, K. (2012) Physics Essays, 25, 488-494. 
https://doi.org/10.4006/0836-1398-25.4.488

[17] Dirac, P.A.M. (1978) Directions in Physics. Wiley, New York, 11. https://doi.org/10.1002/ev.1212

[18] Suto, K. (2014) Applied Physics Research, 6, 64-73. https://doi.org/10.5539/apr.v6n6p64

[19] Suto, K. (2015) Global Journal of Science Frontier Research A, 15, 1-6. https://journalofscience.org/index.php/GJSFR/article/view/1869

[20] Suto, K. (2017) Applied Physics Research, 9, 70-76. https://doi.org/10.5539/apr.v9n1p70

[21] Suto, K. (2017) Journal of Physical Mathematics, 8, 8.

[22] Suto, K. (2020) Journal of High Energy Physics, Gravitation and Cosmology, 6, 52-61. https://doi.org/10.4236/jhepgc.2020.61007 